\title{
Modelos de degradação no pavimento ferroviário a partir de uma revisão sistemática
}

Um dos principais fatores que acelera a degradação do pavimento ferroviário nos últimos anos é o aumento da movimentação de cargas, ocasionado pelas novas demandas exigidas pelo mercado nacional e internacional. Consequentemente, as intervenções de manutenção para correção da geometria da via são desafiadas, exigindo novas técnicas capazes de proporcionar reduções de custos em manutenção e renovação. As empresas ferroviárias no Brasil apresentam valores elevados em gastos com intervenções de manutenções ao longo do trecho concessionado, chegando de $12,4 \%$ na superestrutura e $18,9 \%$ no material rodante. Portanto, o objetivo deste trabalho está voltado em identificar os artigos que apresentem o uso dos modelos de degradação no pavimento ferroviário na literatura científica utilizando a técnica de análise bibliométrica e revisão sistemática, sendo possível verificar o tipo de degradação (geométrico ou estrutural), parâmetros geométricos e os resultados obtidos em cada pesquisa. Observou-se que os modelos de degradação proporcionam a identificação do momento de exigência para aplicação intervenções de manutenção e renovação da via, utilizando as normas técnicas internacionais de índices de qualidade de via ou estado dos componentes ferroviário.

Palavras-chave: Degradação da via; Geometria; Deterioração; Pavimento ferroviário.

\section{Models of degradation in railway pavement from a systematic review}

\begin{abstract}
One of the main factors that accelerates the degradation of the railway pavement in recent years is the increase in cargo handling, caused by the new demands demanded by the national and international market. Consequently, maintenance interventions to correct the track geometry are challenged, requiring new techniques capable of providing cost reductions in maintenance and renovation. Railway companies in Brazil have high amounts of spending on maintenance interventions along the concession stretch, reaching $12.4 \%$ in the superstructure and $18.9 \%$ in the rolling stock. Therefore, the objective of this work is to identify articles that present the use of degradation models on the railway pavement in the scientific literature using the technique of bibliometric analysis and systematic review, making it possible to verify the type of degradation (geometric or structural), parameters and the results obtained in each search. It was observed that the degradation models provide the identification of the moment of demand for application of maintenance and renovation of the track, using the international technical standards of track quality indexes or state of the railway components.
\end{abstract}

Keywords: Track degradation; Geometry; Deterioration; Railway pavement.

Topic: Sustentabilidade nos Transportes

Reviewed anonymously in the process of blind peer.
Received: 06/03/2021

Approved: $24 / 03 / 2021$
Fábio da Silva Martino Fonte

Universidade Federal do Rio de Janeiro, Brasil

http://lattes.cnpq.br/2296562972814690

http://orcid.org/0000-0001-8912-3223

fabiomartino@pet.coppe.ufrj.br

\section{Marina Donato (iD)}

Universidade Federal do Rio de Janeiro, Brasil

http://lattes.cnpq.br/3065688209469583

http://orcid.org/0000-0002-9792-0940

donato@pet.coppe.ufri.br
Referencing this:

FONTE, F. S. M.; DONATO, M.. Modelos de degradação no pavimento ferroviário a partir de uma revisão sistemática. Revista lbero Americana de Ciências Ambientais, v.12, n.3, p.383-397, 2021. DOI: http://doi.org/10.6008/CBPC2179-6858.2021.003.0031 


\section{INTRODUÇÃO}

O aumento de carga nas ferrovias brasileiras nos últimos anos impôs aos gestores de infraestrutura a busca por novos estudos técnicos capazes de gerenciar conflitos em decorrência das novas demandas exigidas pelo mercado nacional e internacional. Segundo o anuário estatístico de transportes realizado pela EPL (2019), na série histórica de movimentação de cargas pelo modo ferroviário foi registrado, do ano de 2010 a 2018, um acréscimo de 129 milhões de TKU, ou seja, em percentual de crescimento de $146 \%$.

O aumento da demanda associado com a limitação de recursos financeiros para investimento em infraestrutura por parte das concessionárias, traz a importância de modelos de degradação da via férrea com foco em otimizar as necessidades de manutenções preventiva e corretiva, reduzir os custos e, consequentemente, melhorar as condições geométricas da via. A previsão e avaliação dos custos de manutenção da via e a seleção das práticas de manutenção mais econômicas são possíveis com um modelo de degradação da via que leva em consideração as principais variações no tráfego, estrutura e parâmetros na manutenção (SADEGHI et al., 2010).

Conseguir manter a qualidade dos componentes da via férrea durante o seu período de ciclo de vida é um desafio permanente para os gestores, tendo como objetivo em manter a geometria da via em perfeitas condições de trafegabilidade. De acordo com condição em que a geometria da via é apresentada, os gestores precisam com antecedência tomar medidas de contingência, como a redução de velocidade, que impacta diretamente na capacidade da via.

Sendo assim, este trabalho tem como objetivo identificar os modelos de degradações sobre o pavimento ferroviário na literatura científica por meio das técnicas de análise bibliométrica e revisão sistemática. Com isso, será possível analisar as tendências dos estudos internacionais voltado à otimização das manutenções na via férrea.

Este artigo está estruturado da seguinte forma. Na seção 2, trata-se de uma breve abordagem sobre os modelos existentes de degradação de via na literatura científica, vale ressaltar que, neste trabalho, serão abordados somente os modelos que se apresentarem na análise bibliométrica. A seção 3 apresenta a metodologia de pesquisa aplicada no estudo e sua importância. Os resultados desta análise bibliométrica serão destacados na seção 4. Por fim, a seção 5 evidencia a conclusão do artigo.

\section{REVISÃO TEÓRICA}

\section{Modelo de degradação da via}

Nos últimos anos, os modelos de degradação da via foram utilizados em vários estudos técnicos, possuindo como objetivo principal a elaboração de condições aplicáveis na manutenção do pavimento ferroviário, principalmente nos países europeus. É possível que tais estudos tenham o intuito de melhorar a qualidade da via, associado as questões ligadas desde a sua geometria até o ciclo de vida dos componentes estruturais do pavimento ferroviário.

O termo pavimento ferroviário foi aplicado pelo professor da COPPE/UFRJ, Jaques Medina, no ano 
de 1988, diante de várias definições relativas aos elementos que compõem a super e infraestrutura. Medina (1988, citado por SILVA, 2002) “propôs instituir-se a terminologia pavimento ferroviário nomeando o sistema estrutural em camadas a ser tratado sob a ótica da Mecânica dos Pavimentos, constituído por trilhos, fixações e demais acessórios metálicos e de apoio, dormentes, lastro, sublastro (quando for o caso) e subleito".

Na revisão bibliométrica, procurou-se analisar e destacar os principais modelos utilizados na literatura internacional. Dentre os modelos destacados por (ELKHOURY et al. 2018), notou-se a utilização dos modelos determinísticos, probabilísticos (Bayesiano e Markov) e estocásticos (Figura 1).

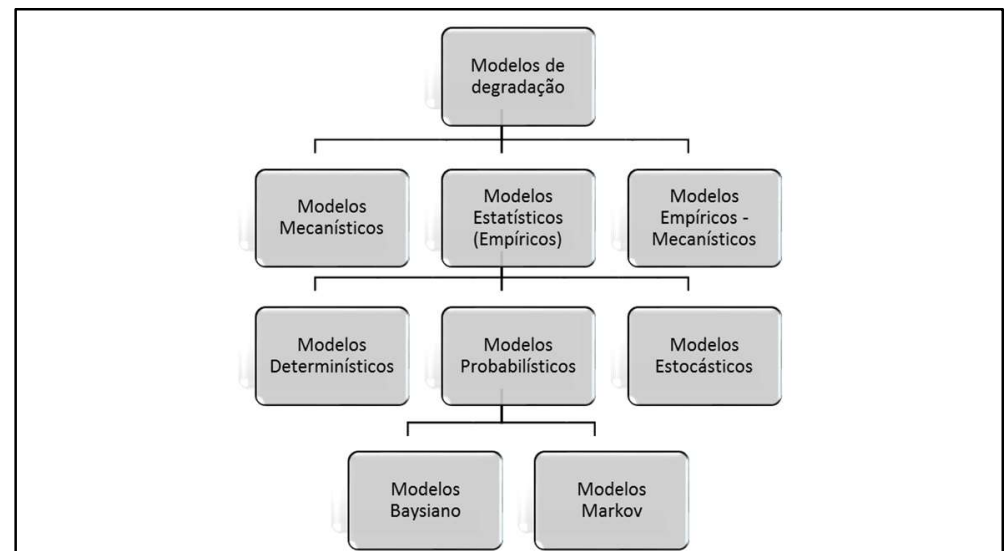

Figura 1: Modelos de degradação de via. Fonte: Modificado de Elkhoury et al. (2018).

Alguns fatores são determinantes para a aceleração da deterioração dos componentes no pavimento ferroviário, destacando: movimentação de cargas (Milhões de toneladas brutas trafegadas - MTBT), velocidade dos trens, tempo entre as atividades de manutenção, colmatação ocasionados finos durante o transporte ou quebra do lastro, temperatura, precipitação de chuvas, curvatura, componentes de baixa qualidade, dentre outros.

Para entendermos melhor cada modelo, será apresentado um breve resumo com as principais características. Percebe-se, ao analisar os artigos selecionados do termo track degradation, um número significativo de autores costuma utilizar os modelos bayesianos a fim de representarem os cálculos matemáticos de degradação da via.

\section{Modelos Estatísticos (Empírico)}

Os modelos estatísticos (Empíricos) necessitam de um banco de dados históricos com os registros das observações sobre a estrutura da via férrea e os fatores que possam ter influência para as possíveis causas de degradação, como tráfego, tipo dos componentes da via e variáveis de manutenção. Ribeiro (2017) destaca que "este tipo de modelo utiliza técnicas de regressão estatística linear ou não linear. As regressões estatísticas são muito utilizadas, por isto, há uma experiência acumulada no seu emprego". Os modelos estatísticos podem ser classificados em três subgrupos: determinísticos, probabilísticos e estocástico.

\section{Modelos Determinísticos}

Modelos determinísticos usam principalmente modelos polinomiais, exponenciais ou lineares de 
vários estágios. Por meio de técnicas de identificação do sistema, é encontrado o conjunto de parâmetros que melhor se ajusta à série de medições (QUIROGA et al., 2011). Este tipo de utiliza expressões matemáticas e estatísticas com o intuito de identificar a relação entre a degradação componentes com a condição em que a via se apresenta.

Para Dahlberg (2001), a união internacional de ferrovias (Internacional Union of Railways - UIC) propôs o modelo determinístico realizado pela ORE (Office for Research and Experiments), com o intuito de estimar a degradação férrea com base nos dados relativo à velocidade da locomotiva, geometria e operação da ferrovia (ou seja, peso por eixo, velocidade da via e volume do tráfego), tonelagem acumulada. A expressão utilizada do modelo determinístico ORE está desenvolvida em:

$$
e=e_{0}+h T^{\alpha}(2 Q)^{\beta} v^{\gamma}
$$

Onde, $e$ é a degradação diretamente após socaria, h é a constante, T é o volume do tráfego, Q é eixo dinâmico, v é a velocidade, e $\alpha, \beta$, e $\gamma$ são parâmetros estimados a partir de dados experimentais.

\section{Modelos Probabilísticos}

De acordo com Elkhoury et al. (2018), vários modelos probabilísticos foram propostos, embora seja difícil analisar os números dos fatores da probabilidade de degradação da via, como o ambiental, os materiais estruturais e a qualidade de construção. Como citado anteriormente, serão apresentadas a seguir as definições dos modelos bayesiano e Markov (Figura 1).

\section{Modelos Bayesiano}

$\mathrm{Na}$ literatura, foram observadas várias equações do modelo bayesiano, destacando o modelo hierárquico bayesiano (HBM), com o principal objetivo de prever a evolução da degradação geométrica da via férrea. Para Andrade et al. (2015), "os modelos bayesianos são diferentes dos modelos estatísticos clássicos, no fato de assumirem parâmetros como variáveis aleatórias, cuja incerteza pode ser quantificada por uma distribuição a priori" .

Da mesma forma, Movaghar et al. (2019) utilizam o modelo bayesiano para analisar a degradação da geometria na rede ferroviária Iraniana, visto que é o melhor para lidar com as incertezas da aplicação dos parâmetros.

Segundo Andrade et al (2012) as abordagens bayesianas divergem da terrorai estatística clássica no fato de que consideram os parâmetros $(\theta)$ como variáveis aleatórias. A distribuição a priori $f(\theta)$ e, então, combinam com a função da verossimilhança $f(x \mid \theta)$ para obter a distribuição a posteriori $f(\theta \mid x)$ diante de um determinado dado observado $(x)$, este tipo de combinação é chamada de regra de Bayes sendo expressa na equação 2:

$$
f(\theta \mid y)=\frac{f(x \mid \theta) \cdot f(\theta)}{f(x)} \propto f(x \mid \theta) \cdot f(\theta)
$$




\section{Modelos Markov}

O modelo de Markov está voltado para a evolução deterioração da via férrea durante as operações de socaria. Elkhoury et al. (2018) relatam que este modelo analisa a taxa de risco da degradação enquanto avaliam a incerteza da degradação da via férrea. Para BAl et al. (2015) utilizam o modelo Markov sobre o gerenciamento das irregularidade e critérios de avaliação estabelecidos pela soma do desvio padrão de sete parâmetros geométricos (Bitola, superelevação, nivelamento direita/esquerda, alinhamento direita/esquerda, torção) com o comprimento de 200 m de seção.

Shafahi et al. (2009) baseia-se na expressão americana Track Quality Index (TQI), para quantificar as irregularidades da via geradas pelas manutenções, sendo coletados por inspeções geométricas (Carro controle). O TQI está estruturado em cinco estados, dentro do intervalo entre 0 a 100, ao qual expressa a probabilidade do possível estado ao longo de um determinado tempo:

$$
p(n)=(p\{X(n)=1\}, p\{X(n)=2\}, \ldots, p\{X(n)=5\})
$$

Onde, $X(n)$ é o estado da via no tempo $n, p\{X(n)=1\}$ é a probabilidade de uma via estar no estado $j$ no tempo $n$.

\section{Modelos Estocásticos}

Os modelos estocásticos são usualmente tratamentos estatísticos baseados em dados históricos, oriundos de inspeções geométricas e visuais. Conforme Andrews et al. (2014), tais modelos são baseados no que realmente aconteceu e explicam a variabilidade por meio do uso da probabilidade, embora, por sua natureza, eles não forneçam percepções sobre a estrutura física.

Lyngby et al. (2008) alegaram que, devido à variabilidade observada, a geometria da via pode ser melhor modelada com um modelo estocástico, os autores utilizam a torção, que é um dos parâmetros de medição da geometria da via, assim como dividiram a deterioração da torção em 50 estados, cada estado representando $1 \mathrm{~mm}$ de torção.

Na análise bibliométrica, observa-se o interesse no desenvolvimento de um modelo que leve em consideração os efeitos da manutenção na geometria da via. Elkhoury et al. (2018) citam que este modelo precisa de mais explicações e compreensão do processo e sugerem mais pesquisas estudos de caso com a aplicação deste modelo.

\section{METODOLOGIA}

Utilizou-se como metodologia a revisão bibliométrica associada a uma revisão sistemática, com foco em analisar os estudos apresentados nos principais periódicos no mundo, além de analisar os objetivos, dados utilizados no modelo, tipo de degradação de via (Geométrico ou estrutural), parâmetros que podem influenciar na degradação da via ou componente ferroviário, além dos resultados.

Araújo (2006) evidencia que, para mensurar, interpretar e avaliar os resultados obtidos das buscas, pesquisadores recorrem a técnicas bibliométricas, que são análises quantitativas com fins a mensurar a produção e disseminação científica. Arantes et al. (2016) complementam que esse tipo de metodologia é recomendado para a análise de portfólios muitos extensos, quando a seleção de maneira quantitativa se 
torna inviável.

Existem três leis que são destacadas sobre a análise bibliométrica por (GUEDES et al., 2005), dentre elas: Lotka, Zipf e Bradford. Sendo que não seja o objetivo deste artigo o aprofundamento das mesmas, apresenta-se abaixo a principal característica relativo a cada lei: I. Lei de Lotka considera que alguns pesquisadores, supostamente de maior prestígio em uma determinada área do conhecimento, produzem muito e muitos pesquisadores, suspostamente de menor prestígio, produzem pouco; II. Lei Zipf permite estimar as frequências de ocorrência das palavras de um determinado texto científico e tecnológico e a região de concentração de termos de indexação, ou palavra-chave, que um pequeno grupo de palavras ocorre muitas vezes e um grande número de palavras é de pequena frequência de ocorrência; III. Lei de Bradford permite estimar o grau de relevância de periódicos em dada área do conhecimento, que os periódicos que produzem o maior número de artigos sobre dado assunto formam um núcleo de periódicos, supostamente de maior qualidade ou relevância para aquela área.

As revisões sistemáticas empregam, portanto, uma metodologia de pesquisa com alto rigor científico, em que se objetiva minimizar o viés da literatura na medida em que é realizada uma recolha exaustiva dos textos publicados sobre o tema que se pretende analisar (CASSUNDÉ et al., 2018).

Sampaio et al. (2007, citado por MENDEIROS et al., 2015) definem a revisão sistemática como sendo um tipo de pesquisa que utiliza como fonte de dados a literatura sobre determinado tema. Esse tipo de investigação disponibiliza um resumo das evidências relacionadas com uma estratégia de intervenção específica, mediante a aplicação de métodos explícitos e sistematizados da busca, apreciação crítica e síntese da informação selecionada.

\section{Coleta de dados (Análise bibliométrica)}

Foram utilizadas as bases de dados Web of Science, Science Direct e Scopus com intuito de analisar o tema abordado. Em primeiro lugar, vale ressaltar o período de pesquisa realizado nas bases de dados dos periódicos feita no mês de abril 2020.

Na primeira etapa do processo, foi inserido a palavra-chave railway infrastructure nas 3 bases de dados, o que resultou em 1.265 artigos. Logo após, na segunda etapa, retirou-se os duplicados utilizando o software Endnote versão $\mathrm{X7}$, identificando 778 artigos. Na terceira etapa do processo, realizou-se o agrupamento das palavras-chave existentes com o intuito de proporcionar uma padronização das demais palavras que apresentaram vários tipos de formatos, dentre elas, algumas estavam no singular e outras no plural. Ao organizar as palavras-chave, notou-se as similaridades e foram distribuídas em grupos. A quarta etapa consiste em analisar os artigos que estão do grupo track degradation.

Após a análise dos artigos neste grupo, observou-se a presença massiva dos termos degradation, geometry e deterioration, sendo que alguns pesquisadores destacaram a utilização dos modelos de degradação, com o foco em otimizar os custos de manutenção voltados para a correção da geometria do pavimento ferroviário. Os termos resultaram em 68 artigos com abordagens de modelos de degradação tanto geométrico como estruturais (Figura 2). 


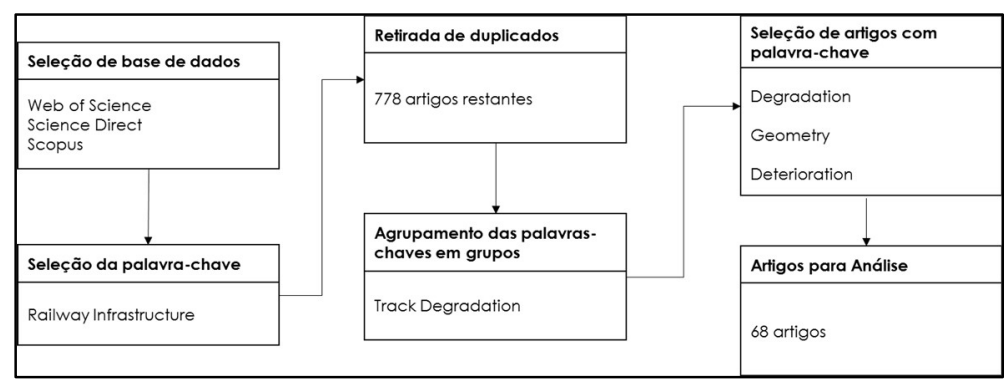

Figura 2: Etapas para obtenção dos artigos para análise.

\section{Análise de dados (Revisão sistemática)}

No caso dos artigos selecionados na análise bibliométrica, não foi realizada a leitura na íntegra de todos por conta da expressiva quantidade. No primeiro instante, é feita da leitura apenas do resumo, da introdução, título e palavras-chave. Neste ponto, vale destacar a importância de elaborar um bom resumo nos artigos científicos, de modo que os leitores tenham informações suficientes para julgar se é conveniente ou não o aprofundamento da leitura.

Após análise dos trabalhos, foram destacados 13 artigos que possuíam a utilização dos modelos de degradação de via na estrutura apresentada por Elkhoury et al. (2018). No capítulo 4, serão ressaltados os principais parâmetros utilizados pelos autores na elaboração de suas pesquisas, assim como a contribuição para a literatura sobre o tema tratado.

\section{RESULTADOS E DISCUSSÃO}

Tabela 1: Classificação dos artigos por modelo, tipo e parâmetros de degradação.

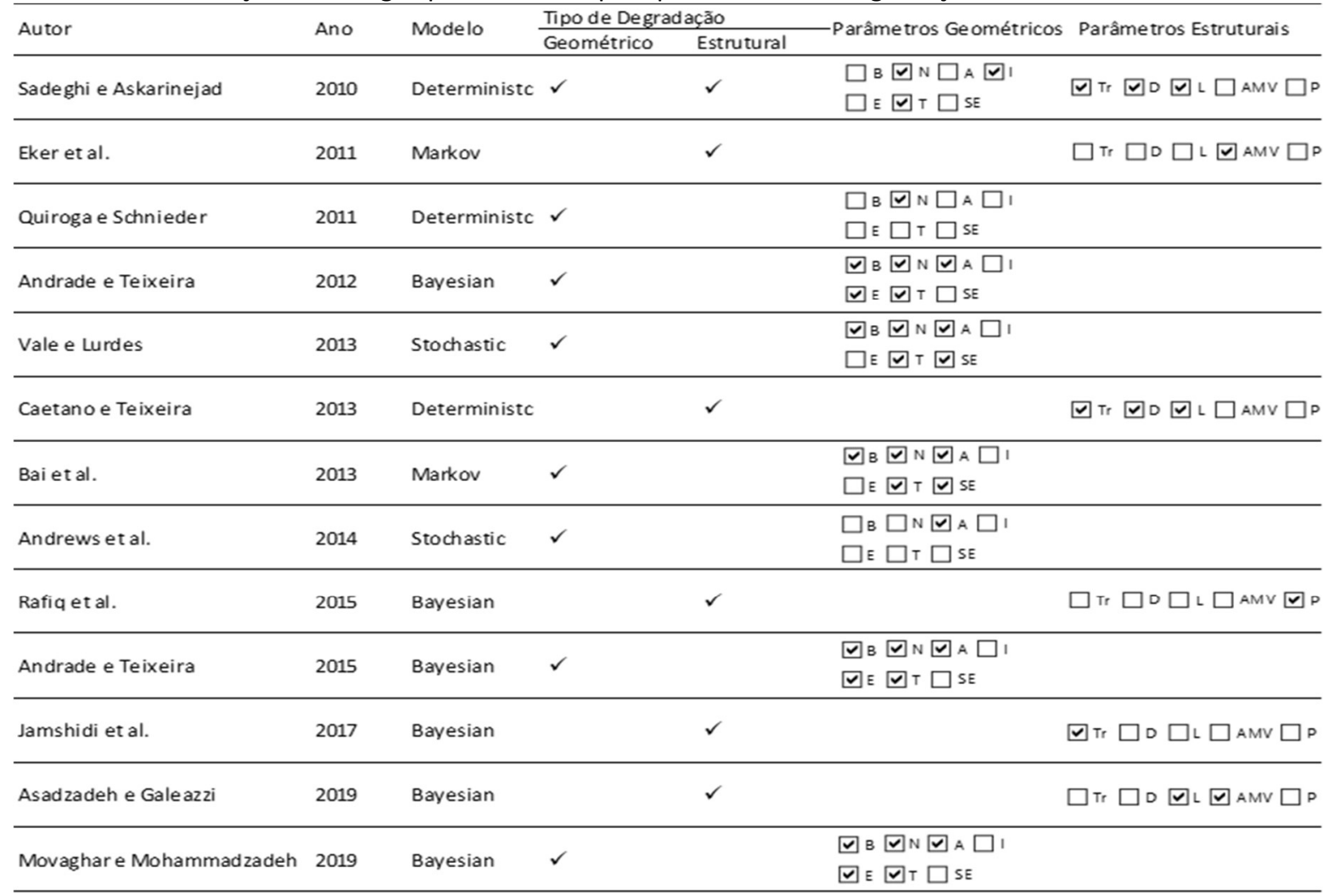

Legenda: $\mathrm{B}=$ Bitola. $\mathrm{N}=$ Nivelamento Longitudinal. $\mathrm{A}=$ Alinhamento. $\mathrm{I}=$ Irregularidades. $\mathrm{E}=$ Empeno. $\mathrm{T}=\mathrm{Torção.} \mathrm{SE}=$ Superelevação. $T r=$ Trilho. $\mathrm{D}=$ Dormente. $\mathrm{L}=$ Lastro. $\mathrm{AMV}=$ Aparelho de mudança de via. $\mathrm{P}=$ Pontes. 
Nesta seção, destaca-se a classificação dos resultados da leitura por parte dos artigos selecionados com a utilização dos modelos de degradação de via. A Tabela 1 apresenta a classificação por modelo, tipo de degradação (Geométrico ou Estrutural) e os parâmetros utilizados. É importante destacar a norma europeia EN 13.848-5 (EN, 2008), que apresenta três limites de alerta recomendados para ações de manutenção preventiva com base em dois indicadores dos parâmetros geométricos mais utilizado no cenário europeu: i) o desvio padrão dos defeitos de nivelamento longitudinal e também ii) o desvio padrão dos defeitos de alinhamento horizontal.

\section{Revisão sistemática dos modelos determinísticos}

A análise bibliométrica indica as pesquisas desenvolvidas por Sadeghi et al. (2010); Quiroga et al. (2011); e C et al. (2013).

O modelo de degradação utilizado por Sadeghi et al. (2010) aborda as limitações e introduz um novo modelo de degradação que permanece simples para os usuários, apesar da incorporação da maioria dos parâmetros nos modelos desenvolvidos anteriormente. Os autores utilizam dois tipos de índices que são uma ramificação do TQI: condições geométricas - Track Geometry Index (TGI) e condições estruturais - Track Structure Index (TSI) Sadeghi et al. (2006, citado por SADEGHI et al., 2010).

O TGI abordada as condições da geometria da via, que influenciam diretamente no conforto da via férrea, sendo que os dados são obtidos pelas inspeções por carro-controle. Os autores utilizam os desvios padrão dos seguintes parâmetros na pesquisa: torção (TI), bitola (GI), alinhamento (Al) e irregularidade (UI):

$$
\mathrm{GI} / \mathrm{TI} / \mathrm{AI} / \mathrm{UI}=100 \exp \left(-\frac{S D_{m}-S D_{n}}{S D_{u}-S D_{n}}\right)
$$

Onde $\mathrm{Gl}, \mathrm{Tl}, \mathrm{Al}$ e $\mathrm{Ul}$ são os parâmetros bitola, torção, alinhamento e irregularidades respectivamente. $\mathrm{SD}_{\mathrm{m}}$ é o desvio padrão do parâmetro medido, $\mathrm{SD}_{n}$ representa o desvio padrão do novo trecho da via e $\mathrm{SD}_{\mathrm{u}}$ São os valores do desvio padrão para uma via que precisa urgentemente de manutenção.

O TSI representa as condições estruturais da via, em que estado se encontram o trilho, dormente, sistema de fixadores, subleito e sistema de drenagem. Sadeghi et al. (2010) utilizam os dados de inspeção visual das linhas ferroviárias, possuindo um banco de dados com os defeitos estruturais reais e aplicando métodos estatísticos. Os autores utilizam 0 para uma linha com um número considerável de defeitos a 100 para uma linha sem nenhum defeito:

$$
(B C I, S C I, R C I)=100-\sum_{i=1}^{p} \sum_{j=1}^{m i} a\left(T_{i}, S_{j}, D_{i j}\right) F(t, d)
$$

onde $\mathrm{BCl}, \mathrm{SCl}$ e $\mathrm{RCl}$ são os índices de condições do lastro, dormente e trilho, respectivamente, a( ) é o valor de ponderação deduzido dependendo do tipo de estresse $\left(T_{i}\right)$, nível de severidade $(S)$ e densidade do estresse $\left(D_{i j}\right), i$ é o contador dos tipos de estresse, $j$ contador do nível de severidade, $p$ número total do tipo de estresse para um componente do estrutura férrea, $m$ é o número do nível de severidade de $i$ e $\mathrm{F}$ ( ) é o fator de ajuste para múltiplos estresses que variam com o valor de dedução total somado $(t)$ e o número individual sobre um valor mínimo estabelecido $(d)$. A quantidade $a(\mathrm{~T}, \mathrm{~S}, \mathrm{D})$ e $\mathrm{F}(t, d)$ pode ser obtida usando equações desenvolvidas no Iranian Railway

Track Maintenance Management System (ITMMS), Sadeghi (2005, citado por SADEGHI et al., 2010). No modelo de degradação utilizado por Quiroga et al. (2011), a deterioração da geometria da via é intensificada após a intervenção de socaria e que precisa ser descrito por uma função exponencial. O modelo desenvolvido é baseado em duas premissas. A primeira é o valor de degradação do nivelamento horizontal 
NLinit $t_{n}$ alcançado após a nth intervenção de socaria. Pode ser descrito por uma variável estocástica distribuída por log-normally, onde $\mu$ é o valor médio e $\sigma^{2}$ é a variância.

$$
\operatorname{NLinit}_{n} \sim \mathcal{L} \mathcal{N}\left(\mu_{N \text { Limit }}(n), \sigma_{N \text { Limit }}^{2}(n)\right)
$$

A evolução do valor de degradação entre duas atividades de socaria pode ser descrita por uma em forma de função exponencial, onde $t$ é o tempo, $t_{n}$ é o tempo da última atividade de socaria, $b_{n}$ é uma variável estocástica distribuída por log-normally e $\varepsilon_{n}(t)$ é a variável distribuída normalmente com valor médio 0.

$$
\operatorname{Ninit}_{n} e^{b_{n}\left(t_{-} t_{n}\right)}+\mathcal{E}(t)
$$

As duas equações precisam ter um banco de dados com medições da geometria da via em diversos setores ferroviários por muitos anos, incluindo as atividades de socaria realizadas. Demonstrando assim a importância de um sistema de gerência de dados que capacite o poder público a analisar de forma simples os dados obtidos pelas concessionárias ferroviárias.

Para caetano et al. (2013), o modelo de degradação foi elaborado para apoio da decisão informada que considere não apenas o custo do ciclo de vida da via férrea (LCC), mas também os tempos de ocupação da via necessários para realizar intervenções. Dentre as várias equações utilizadas pelos autores, destaca-se a função objetiva que consiste na minimização do ciclo de vida total da via férrea:

$$
\begin{aligned}
\min \mathrm{LCC}= & \sum_{\mathrm{w} \in \mathrm{W}} \sum_{\mathrm{t} \in \mathrm{T}} \sum_{\mathrm{n} \in \mathrm{N}} \mathrm{C}_{\mathrm{w}}^{\mathrm{r}} \mathrm{L}_{\mathrm{wtn}} \\
& \quad+\sum_{\mathrm{k} \in \mathrm{K}} \sum_{\mathrm{t} \in \mathrm{T}} \sum_{\mathrm{n} \in \mathrm{N}} \mathrm{C}_{\mathrm{k}}^{\mathrm{m}} \mathrm{X}_{\mathrm{ktn}}-\sum_{w \in W} \sum_{t \in T} \sum_{n \in N} C_{w}^{m} L_{w t n} P_{w t}-\sum_{k \in K} \sum_{n \in N} R_{k n}
\end{aligned}
$$

Onde $K=$ componentes, $N=$ segmento da via, $T=$ tempo da etapa no período do planejamento, $W=$ trabalho de manutenção, $\mathrm{C}_{\mathrm{w}}^{\mathrm{r}}=$ custo unitário do trabalho de renovação, $\mathrm{L}_{\mathrm{wtn}}=$ comprimento da via, $\mathrm{C}_{\mathrm{k}}^{\mathrm{m}}=$ custo de manutenção

para componentes da via, $\mathrm{X}_{\mathrm{ktn}}=$ trabalha com o número de operação manutenção para componente da via, $P_{w t}=$ percentagem custo unitário renovação, $R_{k n}=$ valor residual do componente da via e $C_{w}^{m}=$ custo da manutenção para trabalho de renovação. Por meio da aplicação desta e de outras equações, os autores chegaram ao resultado de que,

quanto maior forem os investimentos em obras de manutenção e renovação, mais serão garantidos os menores índices de indisponibilidade para correções mais severas. Em termos práticos, se houver uma indisponibilidade da via em média de $10 \%$, isso corresponde a aproximadamente $2,4 \mathrm{~h}=$ dia em que a linha ferroviária é ocupada pelas operações M e R.

\section{Revisão sistemática dos modelos Bayesianos}

Os modelos bayesianos apresentaram a maior parte das pesquisas entre os artigos selecionados na análise bibliométrica. Dentre eles, destacam-se: Andrade et al. (2012), Rafiq et al. (2015), Andrade et al. (2015), Jamshidi et al. (2017), Asadzadeh et al. (2019) e Movaghar et al. (2019).

Andrade et al. (2012) destacam na sua pesquisa a equação capaz de melhorar o uso das informações, controle dos processos de manutenção e renovação da via. Os registros de inspeção da via devem ser condensados, geralmente referindo-se às seções da via de 200 metros de comprimento Esveld (1990, citado por ANDRADE et al., 2012). Portanto, a evolução do desvio padrão dos defeitos de nivelamento longitudinal para cada seção de 200 m pode ser estimada usando a seguinte relação linear: 


$$
\sigma_{L D}=C_{1}+C_{0} \cdot T
$$

Onde $\sigma_{L D}$ é o desvio padrão dos defeitos nivelamento longitudinal $(\mathrm{mm}) ; \mathrm{c}_{1}$ é o desvio padrão inicial medido após as operações de renovação e socaria $(\mathrm{mm}) ; c_{0}$ é a taxa de deterioração $(\mathrm{mm} / 100 \mathrm{MGT}) ; \mathrm{T}$ é a tonelagem acumulada desde as operações de renovação ou de socaria (100 MGT). A incerteza associada às taxas de deterioração e ao desvio padrão pode aumentar um pouco quando ocorrerem ações de socaria, mas seu efeito diminuirá mais rápido do que no primeiro ciclo de socaria.

Rafiq et al. (2015) apresentam o desenvolvimento de um modelo de deterioração baseado em condições para tais sistema estrutural usando a metodologia BBN (Bayesian Belief networks), demonstrando as condição geral no grupo dos componentes das pontes ferroviárias e o DBN (Dynamic Bayesian network) sendo estendido para incorporar características dependentes do tempo, a fim de obter perfis de deterioração para o grupo sob diferentes condições de exposição natural, em 50 amostras de pontes ferroviárias no Reino Unido.

Os autores aplicam equações capazes de classificar as condições do deck (Abutment and Wing wall) e da ponte (Barrel arch, face rings, parapets and spandrel wall). Tais valores são classificados em três estados sobre o índice de condição de estruturas, a saber: pobre (intervalo de 0 a 45), regular (intervalo de 46 a 80) e bom (intervalo de 81 a 100). Uma característica destacada da metodologia BBN é sua capacidade de analisar cenários e aplicar 'e se', particularmente útil para a priorização entre a aplicação das atividades de avaliação e de manutenção dos elementos da ponte ferroviária.

O modelo de degradação utilizado por Andrade et al. (2015) possui o objetivo de explorar os modelos hierárquicos bayesianos (HBM) como ferramenta preditiva para avaliar as necessidades de manutenção, dada uma estratégia de manutenção e renovação. Os gerentes de infraestrutura ferroviária na Europa tendem a acionar as ações preventivas de socaria com base em um único indicador: o desvio padrão sobre os defeitos de nivelamento longitudinal, mas a norma europeia EM 13.848-5 recomenda a utilização das ações de manutenção preventiva com base em dois indicadores: i) o desvio padrão dos defeitos de nivelamento longitudinal e também ii) o desvio padrão dos defeitos de alinhamento horizontal.

Os autores evidenciam que o modelo bayesiano difere dos modelos estatísticos clássicos pelo fato de assumirem parâmetros como variáveis aleatórias, cuja a incerteza pode ser quantificada por uma distribuição a priori $\mathrm{p}(\theta)$ e combinada com uma probabilidade de verossimilhança $p(y \mid \theta)$, com isto obtém a distribuição a posteriori $\mathrm{p}(\theta \mid \mathrm{y})$ dos parâmetros de interesse e sobre os dados observados $y$, podendo assim ser calculada de acordo com a regra de Bayes, já citada na equação 2 .

Jamshidi et al. (2017) fazem abordagem do risco de quebra do trilho com base em uma estrutura integrada que mescla as informações de duas variáveis relacionadas aos defeitos: comprimento visual e crescimento de trincas. Sendo assim, utilizaram o modelo de inferência bayesiana para estimar a probabilidade da falha no trilho. A função de crescimento de trinca e a função de gravidade parcialmente inversa:

$$
\text { Risk } \propto \text { Fprob }\left(F_{C}\left(F_{S, i n v .}\left(V_{1}, V_{2}\right)\right)\right)
$$

Onde $V_{1}$ e $V_{2}$ são duas medições consecutivas de inspeções visuais para um defeito detectado por análise de dados de imagens, e $F_{S}$, inv. relaciona $V_{1}$ e $V_{2}$ com milhões de toneladas brutas (MTB). A função $F_{C}$ relaciona MTB ao crescimento da trinca e a função $F_{\text {prob }}$ estima a probabilidade de falha com base na 
estimativa do crescimento da trinca.

No estudo de caso aplicado na ferrovia ao norte da Holanda por Jamshidi et al. (2017), o defeito squats no trilho salientou o estágio grave (severe) na análise de severidade, sendo sujeito a quebra do trilho caso não ocorra nenhuma operação de manutenção preventiva dentro de uma determinada etapa do MBT.

Asadzadeh et al. (2019) desenvolveram uma metodologia integrada (método de análise fractal com modelagem probabilística e inferência Bayesiana) capaz de realizar uma previsão da taxa de degradação de lastro nos aparelhos de mudança de via (AMV), as taxas de irregularidades da via foram geradas por meio do carregamento cumulativo da via. Os autores utilizam a atualização Bayesiana sendo importante na análise dinâmica de uma sequência de dados e para o cálculo da taxa de degradação do lastro (Ballast degradation rate $-B D R)$.

Vale ressaltar que Asadzadeh et al. (2019) utilizaram dados históricos de carro-controle em 15 AMVs na linha ferroviária dinamarquesa, velocidade da via, tonelagem acumulada, direção, número de socaria, temperatura do ar, precipitação, alinhamento da via, torção, bitola, nivelamento longitudinal. Estes fatores foram agrupados nos seguintes grupos: i) fatores relativos à geometria; ii) Fatores relativos ao tráfego; iii) fatores relativos à manutenção; iv) fatores relativos ao clima. $\mathrm{O}$ estudo mostrou que o BDR nos aparelhos de mudança de via é fortemente afetado por fatores relacionados à geometria da via (Alinhamento, bitola, nível longitudinal, torção e escala) e ao clima (Temperatura e precipitação da chuva), juntos podem explicar cerca de $52 \%$ de variação no BDR.

Movaghar et al. (2019) explicitaram no TQI a incerteza quanto à classificação da qualidade ainda existente após a observação dos dados na rede ferroviária iraniana. Os autores estruturam a análise bayesiana da seguinte forma: i) modelo de probabilidade $p\left(y_{i} \mid \theta\right)$; ii) distribuição a priori $p(\theta)$; iii) função de verossimilhança $L(\theta \mid y)$; e iv) distribuição a posteriori $p(\theta \mid y)$. Sendo utilizado a EN 13848-5 que define o desvio padrão do nivelamento longitudinal e alinhamento como limite de alerta, com isto, criaram três classes de qualidades diferentes $Q_{1} a_{3} Q_{3}$ Quanto menor o desvio padrão, melhor a condição da via, com isto, o gerente de infraestrutura tenta manter a qualidade da via na classe $Q_{1}$.

Foram divididos em 13 blocos de seções tangentes de 200 m, os resultados da aplicação do modelo chegaram a 77\% apresentando o nível de qualidade Q3, bem como apenas $8 \%$ dos blocos não precisam de ações de manutenção. Mesmo que a maioria dos blocos seja classificado na classe Q3, esses resultados são adequados para priorizar as ações de manutenção.

\section{Revisão sistemática dos modelos Markov}

Nas pesquisas utilizando as formulações de Markov, destacam-se: Eker et al. (2011) e Vai et al. (2015). Eker et al. (2011) utiliza-se o método simples de prognóstico baseado no estado (Simple State Based Prognostic - SSBP) com o intuito de detectar e prever a progressão de falhas nos sistemas eletromecânicos em AMVs, assim como comparando com os modelos ocultos de Markov (Hidden Markov Models - HMMs). Os dados foram coletados por sensores com capacidade para medir as tensões aplicadas nos aparelhos eletromecânicos e, consequentemente, analisar a degradação destes aparelhos, que possuem como uma das 
funções movimentar as agulhas do AMVs.

Nesta pesquisa, foi empregado a Hierarchical Hidden Markov Model (HHMM), que é uma versão hierárquica de um HMM projetado para modelar estruturas hierárquicas de dados sequencias. EKER et al. (2011) identificam o estado da camada oculta superior e inferior do AMV por um determinado período de tempo. A camada oculta superior representa os estados de integridade do sistema de AMV, enquanto os estados da camada oculta inferior representam o estado do movimento dos trilhos, ou seja, a localização durante o movimento de ida e volta do trilho. Em outras palavras, a estrutura hierárquica pode ser mantida de tal forma que o 'nível superior' não pode mudar de estado, a menos que o estado de 'nível inferior' atinja o último estado possível.

Por fim, os autores concluem que o método SSBP é muito simples e supera os prognósticos baseados em HHMM, uma vez que as falhas geralmente passam por vários estados antes de figurar como um sistema inutilizável. Como desvantagem, o método apresentado possui restrições nas transições de estado que permitem a transição de um estado apenas para ele mesmo e um estado consecutivo.

Bai et al. (2015) aplicam a teoria do processo estocástico de Markov que é utilizada para investigar a deterioração entre duas atividades consecutivas de manutenções devido às irregularidades da via. Os autores usam o TQI para quantificar as irregularidades da via nas unidades de manutenção. Foram considerados na ferrovia Chinesa seções de 200 m como unidade de cálculo do desvio padrão de setes parâmetros geométricos: bitola, torção, superelevação, alinhamento (direita e esquerda), nivelamento longitudinal (direita e esquerda).

A probabilidade de transição de Markov descreve as incertezas dos estados em que se apresentam as irregularidades da geometria da via entre o intervalo de duas atividades de inspeção. $O t_{\mathrm{A}}$ e $t_{\mathrm{B}}$ representam duas datas de inspeções consecutivas para a unidade de manutenção da via, $h\left(t_{A}\right)$ expressa o estado irregular no tempo $t_{\mathrm{A}}$, tão $h\left(t_{\mathrm{A}}\right)=i(i=1,2,3,4) \cdot h\left(t_{\mathrm{B}}\right)$ expressa o estado irregular no tempo $t_{\mathrm{B}}$, tão $h\left(t_{\mathrm{B}}\right)=i(i=1,2,3,4), i$ é o estado de irregularidade da via no tempo $t_{\mathrm{A}}$ e $j$ irregularidade do estado da via no tempo $t_{\mathrm{B}}$. $\mathrm{A}$ probabilidade pode ser expressa como:

$$
\begin{gathered}
\operatorname{prob}\left(h\left(t_{B}\right)=j \mid h\left(t_{A}\right)=i\right)=\pi_{i j} \\
\pi_{i j} \geq 0 \quad \pi_{i j}=0 \text { quando } \mathrm{i}>\mathrm{j} \quad \sum_{j=1}^{J} \pi_{i j}=1
\end{gathered}
$$

Onde:

Após a aplicação das equações, os autores concluíram que os fatores heterogêneos desempenham um papel significativo nas taxas de deterioração. Vale destacar que os fatores que influenciam no processo de deterioração de uma unidade de manutenção da via foram classificados: i) fatores de transportes (como tonelagem bruta acumulada e velocidades do trem); ii) calendário das atividades de manutenção da infraestrutura; iii) fatores naturais (como temperatura, clima e areia); iv) fatores de instalação associadas à via (como eficácia do dormente, limpeza dos lastros e condição subleito); v) fatores de condição da ferrovia (curvas de pequeno raio, grandes rampas longas e túneis); e vi) fatores de condição de transportes (como 
transporte a granel ou em contêineres de carvão, areia e minerais). As unidades de manutenção da via tinham a mesma quilometragem da amostra, no entanto, uma se deteriorou mais rápido do que a outra por causa de seus diferentes fatores heterogêneos.

\section{Revisão sistemática dos modelos Estocásticos}

Nota-se que os modelos estocásticos estiveram presentes em duas pesquisas durante a análise bibliométrica com a utilização de modelo de degradação, sendo Vale et al. (2013) e Andrews et al. (2014).

Vale et al. (2013) destacam um modelo estocástico para caracterizar o processo de degradação geométrico da via ao longo do tempo. Aplicam o estudo de caso na ferrovia do norte de Portugal, ligando Lisboa a Porto. O intervalo de tempo entre as inspeções dinâmicas corresponde cerca de 90 dias. Dentre estas inspeções de geometria, 5 parâmetros são medidos pelo carro-controle: o nivelamento longitudinal, alinhamento, superelevação, bitola e torção.

Os autores utilizam a distribuição Dagum, usualmente adotada para representar o processo de degradação geométrica da via em termos do nivelamento horizontal. O modelo em análise destaca os três parâmetros de distribuição de Dagum, cuja função $F(x)$, é definida por:

$$
F(x)=\left[1+\left(\frac{x}{\beta}\right)^{-\alpha}\right]^{-k}, \mathrm{x}>0
$$

Onde $\alpha, \beta$ e k são parâmetros positivos e $\mathrm{x}$ uma variável aleatória. Os resultados demonstraram que o parâmetro $\beta$ depende linearmente da taxa média de degradação do desvio padrão do nivelamento longitudinal, sendo menor para velocidades máximas admissíveis do veículo.

Andrews et al. (2014) desenvolvem a base de um modelo de caso que analisa uma potencial estratégia de gerenciamento de ativos da via. Possui a formulação estocástica Petri net (PN), levando em consideração os processos de degradação, inspeção, reparo e renovação da geometria. Usando o modelo, os efeitos das mudanças na estratégia de manutenção podem ser avaliados e a opção mais eficaz selecionada a fim de reduzir os custos de toda a vida ao mesmo tempo em que fornece um nível de desempenho aceitável.

Os autores citam que a característica que melhor indica a necessidade de aprimorar a geometria é o desvio padrão do parâmetro de alinhamento vertical. A análise das intervenções de socaria é associada a dois parâmetros de distribuição Weibull com parâmetros $\beta$, o fator de forma, e $\eta$, característica de vida, a distribuição de tempo para deteriorar para qualquer desvio padrão especificado pode ser determinada.

Logo após, Andrews et al. (2014) dividem os resultados em três históricos de manutenção, sendo representado pelas intervenções de inspeções, reparação e renovação. Na questão de inspeção, verifica-se, à medida que as inspeções geométricas aumentam, a proporção de manutenção realizada nas condições mais precárias da via aumenta. Conforme os tempos de manutenções de rotina aumentam, uma porcentagem maior de tempo é gasto na via e a condição conhecida por exigir manutenção. Caso reduza o tempo médio de reparo de rotina para 50 dias, pode proporcionar um excelente desempenho com a via em boas condições $98,688 \%$ do tempo.

Por fim, referente a renovação, foi aplicado para 20 e 40 anos para cada seção da via, gerando em média 2,29 intervenções para a renovação de 20 anos e 4,34 intervenções para o cenário de 40 anos. 


\section{CONCLUSÕES}

O estudo destacou os modelos de degradações no pavimento ferroviário presentes na literatura científica internacional utilizando as técnicas de análise bibliométrica e revisão sistemática dos artigos que aplicam estes modelos. A análise bibliométrica trouxe à tona debate sobre uma das maiores preocupações por parte dos gerentes de infraestrutura e poder público de vários países, a importância sobre a qualidade geométrica e estrutural da ferrovia, tanto de cargas quanto para passageiros. A revisão sistemática proporcionou o aprofundamento dos estudos sobre a colaboração científica quando foram tratados os parâmetros utilizados e os resultados obtidos.

Dentro os modelos destacados nesta análise, demonstra-se predominância pelos estudos Bayesianos. Tal modelo proporciona a comparação dos diferentes parâmetros de qualidade da via abordados pela ORE. Assim, aplica-se ao sistema de alerta capaz de informar aos tomadores de decisões sobre o momento econômico para a realização de uma manutenção ou renovação do trecho da via.

Um ponto a ser destacado durante a análise bibliométrica do termo 'track degradation' diz respeito ao fato de que todos os estudos técnicos foram aplicados em países da Europa, Ásia e Oriente médio. Na américa latina, não ocorreu a publicação acerca da elaboração de um trabalho prático utilizando os referidos índices de qualidade da via. Uma das possíveis impedâncias para realização deste tipo de pesquisa seria a disponibilização de inspeções geométricas por carro-controle ou outras tecnologias por parte das concessionárias brasileiras e poder público, visto que este tipo de inspeção poderia proporcionar a real situação da geometria da via.

Por isto, sugere-se a aplicação destes modelos de degradação no cenário brasileiro, pois o pavimento ferroviário seja mais impactado pelas grandes movimentações de cargas (minerais, granéis, commodities, dentre outros), gerando, assim, gastos em manutenção e renovação dos componentes da via. Outro ponto seria a elaboração de um sistema inteligente sobre a gerência do pavimento ferroviário capaz de absorver dados geométricos, estruturais e os seis fatores (BAl et al., 2015).

\section{REFERÊNCIAS}

ANDRADE, A. R.; TEIXEIRA, P. F.. A Bayesian model to assess rail track geometry degradation through its life-cycle. Research in Transportation Economics, v.36, n.1, p.1-8, 2012. DOI: http://doi.org/10.1016/j.retrec.2012.03.011

ANDRADE, A. R.; TEIXEIRA, P. F.. Statistical modelling of railway track geometry degradation using Hierarchical Bayesian models. Reliability Engineering and System Safety, v.142, p.169-183, 2015. DOI:

http://doi.org/10.1016/j.ress.2015.05.009

ANDREWS, J.; PRESCOTT, D.; ROZIÈRES, F.. A stochastic model for railway track asset management. Reliability Engineering and System Safety, v.130, p.76-84, 2014. DOI: http://doi.org/10.1016/j.ress.2014.04.021

ARANTES, F. P.; SANTOS, T. F.. Integração da cadeia de suprimentos: uma análise teórica e bibliométrica das publicações. In: ENCONTRO NACIONAL DE ENGENHARIA DE
PRODUÇÃO, 36. Anais. 2016.

ARAÚJO, C. A.. Bibliometria: evolução história e questões atuais. Em questão, v.12, n.1, p.11-32, 2006.

ASADZADEH, S. M.; GALEAZZI, R.. An integrated methodology for the prognosis of ballast degradation in railway turnouts. Proceedings of the Institution of Mechanical Engineers, Part F: Journal of Rail and Rapid Transit, v.234, n.8, 2019. DOI: http://doi.org/10.1177/0954409719878614

BAI, L.; LIU, R.; SUN, Q.; WANG, F.; XU, P.. Markov-based model for the prediction of railway track irregularities. Proceedings of the Institution of Mechanical Engineers, Part F: Journal of Rail and Rapid Transit, v.229, n.2, p.150159, 2015. DOI: http://doi.org/10.1177/0954409713503460

CAETANO, L. F.; TEIXEIRA, P. F.. Availability approach to 
optimizing railway track renewal operations. Journal of Transportation Engineering, v.139, n.9, p.941-948, 2013. DOI: http://doi.org/10.1061/(ASCE)TE.1943-5436.0000575

CASSUNDÉ, F. R. S. A.; BARBOSA, M. A. C.; MENDONÇA, J. R. C.. Entre revisões sistemáticas e bibliometrias: Como tem sido mapeada a produção acadêmica em administração no Brasil?. Inf. Londrina, v.23, n.1, p.311-334, 2018.

DAHLBERG, T.. Some railroad settlement models: a critical review. Proceedings of the Institution of Mechanical Engineers, Part F: Journal of Rail and Rapid Transit, v.215, n.4, p.289-300, 2001. DOI: http://doi.org/10.1243/0954409011531585

EKER, O. F.; CAMCI, F.; GUCLU, A.; YILBOGA, H.; SEVKLI, M.; BASKAN, S.. A simple state-based prognostic model for railway turnout systems. leee Transactions on Industrial Electronics, v.58, n.5, p.1718-1726, 2011. DOI: http://doi.org/10.1109/TIE.2010.2051399

ELKHOURY, N.; HITIHAMILLAGE, L.; MORIDPOUR, S.; ROBERT, D.. Degradation prediction of rail tracks: A review of the existing literature. Open Transportation Journal, v.12, p.88-104, 2018. DOI:

http://doi.org/10.2174/1874447801812010088

EN. European standard EN 13848-5: Railway applications Track - Track geometry quality - Part 5: Geometric quality levels. European Commitee for Standardization (CEN), 2008.

EPL. Boletim de Logística. Observatório Nacional de Transporte e Logística - ONTL, Empresa de Planejamento e Logística S.A. Brasília: EPL, 2019.

GUEDES, V. V.; BORSCHIVER, S.. Bibliometria: uma ferramenta estatística para a gestão da informação e do conhecimento, em sistemas de informação, de comunicação e de avaliação científica e tecnológica. In: ENCONTRO NACIONAL DE CIÊNCIAS DA INFORMAÇÃO. Anais. 2005.

JAMSHIDI, A.; FAGHIH-ROOHI, S.; HAJIZADEH, S.; NUNEZ, A.; BABUSKA, R.; DOLLEVOET, R.; LI, Z. L.; SCHUTTER, B.. A Big Data Analysis Approach for Rail Failure Risk Assessment. Risk Analysis, v.37, n.8, p.1495-1507, 2017. DOI: http://doi.org/10.1111/risa.12836

LYNGBY, N.; HOKSTAD, P.; VATN, J.. RAMS Management of Railway Tracks. Handbook of Performability Engineering, p.1123-1145, 2008. DOI: http://doi.org/10.1007/978-184800-131-2 68
MEDINA, J. D.. Fundamentos da Mecânica dos Pavimentos. Rio de Janeiro: COPPE, 1988.

MENDEIROS, I. I.; VIEIRA, A.; BRAVIANO, G.; GONÇALVES, B. S.. Revisão sistemática e bibliometria facilitadas por um Canvas para visualização de informação. Revista Brasileira de Design da Informação, v.12, n.1, p.93-110, 2015.

MOVAGHAR, M.; MOHAMMADZADEH, S.. Intelligent index for railway track quality evaluation based on Bayesian approaches. Structure and Infrastructure Engineering, v.16, n.7, 2019. DOI:

http://doi.org/10.1080/15732479.2019.1676793

QUIROGA, L. M.; SCHNIEDER, E.. Monte Carlo simulation of railway track geometry deterioration and restoration. Proceedings of the Institution of Mechanical Engineers, Part O: Journal of Risk and Reliability, v.226, n.3, p.274-282, 2011. DOI: http://doi.org/10.1177/1748006X11418422

RAFIQ, M. I.; CHRYSSANTHOPOULOS, M. K.; SATHANANTHAN, S.. Bridge condition modelling and prediction using dynamic Bayesian belief networks. Structure and Infrastructure Engineering, v.11, n.1, p.38-50, 2015. DOI: http://doi.org/10.1080/15732479.2013.879319

RIBEIRO, F. S.. Contribuição para análise do custo do ciclo de vida de um sistema de gerência de pavimento ferroviário. Tese (Doutorado) - Escola Politécnica da Universidade de São Paulo, São Paulo, 2017.

SADEGHI, J.; ASKARINEJAD, H.. Development of improved railway track degradation models. Structural and Infrastructure Engineering, v.6, n.6, p.675-688, 2010.

SHAFAHI, Y.; HAKHAMANESHI, R.. Application of a maintenance management model for Iranian Railways based on the Markov chain and probabilistic dynamic programming. Scientia Iranica, v.16, n.1A, p.87-97, 2009.

SILVA, L. F. M.. Fundamentos teórico-experimentais da mecânica dos pavimentos ferroviários e esboço de um sistema de gerência aplicado à manutenção da via permanente. Rio de Janeiro: COPPE, 2002.

VALE, C.; LURDES, S. M.. Stochastic model for the geometrical rail track degradation process in the Portuguese railway Northern Line. Reliability Engineering \& System Safety, v.116, p.91-98, 2013. DOI: http://doi.org/10.1016/j.ress.2013.02.010

A CBPC - Companhia Brasileira de Produção Científica (CNPJ: 11.221.422/0001-03) detém os direitos materiais desta publicação. Os direitos referem-se à publicação do trabalho em qualquer parte do mundo, incluindo os direitos às renovações, expansões e disseminações da contribuição, bem como outros direitos subsidiários. Todos os trabalhos publicados eletronicamente poderão posteriormente ser publicados em coletâneas impressas sob coordenação da Sustenere Publishing, da Companhia Brasileira de Produção Científica e seus parceiros autorizados. Os (as) autores (as) preservam os direitos autorais, mas não têm permissão para a publicação da contribuição em outro meio, impresso ou digital, em português ou em tradução. 